CUBO A Mathematical Journal

Vol.19, $N^{\underline{O}} 01$, (39-51). March 2017

\title{
Maximal functions and properties of the weighted composition operators acting on the Korenblum, $\alpha$-Bloch and $\alpha$-Zygmund spaces
}

\author{
Gabriel M. Antón Marval ${ }^{1}$, René E. Castillo ${ }^{2}$, Julio C. Ramos-Fernández ${ }^{3}$ \\ 1 Area de Matemáticas, Universidad Nacional Experimental de Guayana, \\ Puerto Ordaz 8050, Estado Bolívar, Venezuela. \\ ${ }^{2}$ Departamento de Matemáticas, Universidad Nacional de Colombia, \\ AP360354 Bogotá, Colombia. \\ 3 Departamento de Matemáticas, Universidad de Oriente, \\ Cumaná 6101, Estado Sucre, Venezuela. \\ gabman@gmail.com, recastillo@unal.edu.co, jcramos@udo.edu.ve
}

\begin{abstract}
Using certain maximal analytic functions, we obtain new characterizations of the continuity and compactness of the weighted composition operators when acts between Korenblum spaces, $\alpha$-Bloch spaces and when acts from certain weighted Banach spaces of analytic functions with a logarithmic weight into $\alpha$-Bloch spaces. As consequence of our results, we obtain a new characterization of the continuity and compactness of composition operators acting between $\alpha$-Zygmund spaces.
\end{abstract}

\section{RESUMEN}

Usando ciertas funciones analíticas maximales, obtenemos nuevas caracterizaciones de la continuidad y compacidad de operadores de composición con pesos cuando actúan entre espacios de Korenblum, espacios $\alpha$-Bloch y cuando actúan desde ciertos espacios de Banach de funciones analíticas con un peso logarítmico en espacios $\alpha$-Bloch. Como consecuencia de nuestros resultados, obtenemos una nueva caracterización de la continuidad y la compacidad de operadores de composición actuando entre espacios $\alpha$-Zygmund.

Keywords and Phrases: Weighted Banach spaces of analytic functions, Bloch space, weighted composition operators.

2010 AMS Mathematics Subject Classification: 30D45, 47B33. 


\section{Introduction}

Over recent years, there has been a growing interest in the study of the properties of the weighted composition operators when acts between Banach spaces of analytic functions on $\mathbb{D}$, the open unit disk of the complex plane $\mathbb{C}$. For fixed holomorphic functions $u: \mathbb{D} \rightarrow \mathbb{C}$ and $\phi: \mathbb{D} \rightarrow \mathbb{D}$, we can define the linear operator $W_{\mathfrak{u}, \phi}: H(\mathbb{D}) \rightarrow H(\mathbb{D})$, where $H(\mathbb{D})$ denotes the space of all holomorphic functions, by

$$
W_{u, \phi}(f):=u \cdot(f \circ \phi) .
$$

Which is known as the weighted composition operator with symbols $u$ and $\phi$. Clearly, if $u \equiv 1$ we have $W_{1, \phi}(f)=f \circ \phi=C_{\phi}(f)$, the composition operator $C_{\phi}$, and if $\phi(z)=i d(z)=z$ for all $z \in \mathbb{D}$, we obtain $W_{u, i d}(f)=u \cdot f=M_{\mathfrak{u}}(f)$, the multiplication operator $M_{\mathfrak{u}}$. Furthermore, we can see that $W_{\mathfrak{u}, \phi}$ is $1-1$ on $H(\mathbb{D})$ unless that $u \equiv 0$ or $\phi$ is a constant function.

Montes-Rodríguez in [16] and Contreras and Hernández-Díaz in [9] characterized the continuity, the compactness and calculated the essential norm of $\mathrm{W}_{\mathfrak{u}, \phi}: \mathrm{H}_{v}^{\infty} \rightarrow \mathrm{H}_{w}^{\infty}$ in terms of a quotient involving the associated weight of $v$, under the requirement that the weights (a weight function is a bounded, continuous and positive function defined on $\mathbb{D}) v$ and $w$ are radial $(v(|z|)=v(z)$ for all $z \in \mathbb{D}$ ), non-increasing (i.e. $v\left(r_{1}\right) \geq v\left(r_{2}\right)$ for all $0<r_{1}<r_{2}<1$ ) and typical (that is, $\left.\lim _{|z| \rightarrow 1^{-}} v(z)=0\right)$. Here, $H_{v}^{\infty}$ is the weighted Banach spaces of analytic functions or growth spaces which consist of functions $f \in H(\mathbb{D})$ such that

$$
\|\mathrm{f}\|_{H_{v}^{\infty}}=\sup _{z \in \mathbb{D}} v(z)|f(z)|<\infty .
$$

The associated weight of $v$, denoted by $\widetilde{v}$, is defined by

$$
\widetilde{v}(z)=\left(\sup _{\|f\|_{H_{v}^{\infty}} \leq 1}\{|f(z)|\}\right)^{-1}, \quad z \in \mathbb{D} .
$$

The weighted Banach spaces of analytic functions or growth spaces $\mathrm{H}_{v}^{\infty}$ are natural generalizations of $H^{\infty}$, the space of all bounded analytic functions on the open unit disk $\mathbb{D}$. It is known that $\mathrm{H}_{v}^{\infty}$ is a Banach space with the norm defined in (1.1). Initially, interest in the weighted Banach spaces of analytic functions were oriented to studying the growth conditions of analytic functions and the duality of these spaces, being present in various areas such as complex analysis, Fourier analysis, spectral theory and partial differential equations. Some examples may be found in [3]. The associated weight $\widetilde{v}$ of $v$ was introduced by Anderson and Duncan in [1] and studied by Bierstedt, Bonet and Taskinen in [2]. The associated weight is a very important tool in the study of the weighted Banach spaces of analytic functions, it is known that the space $\mathrm{H}_{\widetilde{v}}^{\infty}$ is isometrically equal to $\mathrm{H}_{v}^{\infty}$, that is $\|\mathrm{f}\|_{\mathrm{H}_{\widetilde{\infty}}^{\infty}}=\|\mathrm{f}\|_{\mathrm{H}_{v}^{\infty}}$ for all $\mathrm{f} \in \mathrm{H}_{v}^{\infty}$ and $v$ is dominated by its associated weight, that is, $\widetilde{v} \geq v>0$ on $\mathbb{D}$. We said that a weight $v$ is essential if $\widetilde{v} \sim v$, that is, if there is a constant $\mathrm{C}_{v}>0$ such that $v(z) \leq \widetilde{v}(z) \leq \mathrm{C}_{v} v(z)$ for all $z \in \mathbb{D}$. We refer to the interested reader the works [2] and [4] for more properties of the associated weight and the space $\mathrm{H}_{v}^{\infty}$. 
The Korenblum's spaces (also known as Bergman spaces) $\mathrm{H}_{\alpha}^{\infty}$ are particular cases of the growth spaces, they are obtained when the weights are the functions $v_{\alpha}(z)=\left(1-|z|^{2}\right)^{\alpha}$ with $z \in \mathbb{D}$, where the parameter $\alpha$ is positive and fixed. Clearly the weight $v_{\alpha}$ is radial, typical, non-increasing and essential. We refer to [12] for more details about the Korenblum's spaces.

The Bloch-type space $\mathcal{B}^{v}$ are related with the growth spaces, consist of all analytic function $f$ on $\mathbb{D}$ such that $\mathrm{f}^{\prime} \in \mathrm{H}_{v}^{\infty}$. $\mathcal{B}^{v}$ is a Banach space with the norm

$$
\|\mathbf{f}\|_{\mathcal{B}^{v}}=|\mathbf{f}(0)|+\left\|\mathbf{f}^{\prime}\right\|_{H_{v}^{\infty}}=|\mathbf{f}(0)|+\|\mathbf{f}\|_{\widetilde{\mathcal{B}}^{v}}
$$

where,

$$
\|f\|_{\widetilde{\mathcal{B}}^{v}}=\sup _{z \in \mathbb{D}} v(z)\left|f^{\prime}(z)\right| .
$$

When $v(z)=1-|z|^{2}$, the space $\mathcal{B}^{v}$ becomes the classical Bloch space and it is denoted by $\mathcal{B}$, we refer to [23] for more details about Bloch's space. When $v(z)=\left(1-|z|^{2}\right)^{\alpha}$, where $\alpha>0$ is fixed, we get the $\alpha$-Bloch spaces which is denoted by $\mathcal{B}^{\alpha}$ (see [24]).

The study of the properties of composition operators on Bloch-type spaces began with the celebrated work of Madigan and Matheson in [14], they characterized continuity and compactness for composition operators on the classical Bloch space $\mathcal{B}$. Their results have been extended by a big numerous of authors (see [18] and the lot of references therein). However, the properties of the weighted composition operators acting on Bloch-type space is still in development and there is not much references about this subject, it is remarkable the work of Ohno, Stroethoff and Zhao [17], where they characterized the continuity and the compactness of the weighted composition operators acting between $\alpha$-Bloch spaces in terms of the continuity and compactness of certain weighted composition operators acting between certain growth spaces.

In [22], Tjani shows that the composition operator $\mathrm{C}_{\phi}: \mathcal{B} \rightarrow \mathcal{B}$ is compact if and only if $\left\|C_{\phi}\left(\alpha_{\lambda}\right)\right\|_{\mathcal{B}} \rightarrow 0$ as $|\lambda| \rightarrow 1$, where $\alpha_{\lambda}(z)=(\lambda-z) /(1-\bar{\lambda} z)$ is the Mobius transformation of the unit disk. A similar result for weighted composition operators from Hardy spaces into logarithmic Bloch spaces was obtained by Colonna and Li in [8]. Also, Giménez, Malavé and Ramos-Fernández in [11] extended the Tjani's result to composition operators $C_{\phi}: \mathcal{B} \rightarrow \mathcal{B}^{\mu}$, where the weight $\mu$ was taken to be a non-vanishing, complex valued holomorphic function satisfying a reasonable geometric condition on the Euclidean disk $\mathrm{D}(1,1)$. More recently, by changing the functions $\alpha_{\lambda}$, used by Tjani, to certain maximal analytic functions $\sigma_{a}$ with $a \in \mathbb{D}$, Ramos-Fernández et. al. $[15,19,6,5]$ show that Tjani's result can be extended to the case of composition operators acting on $\alpha$-Bloch and weighted Bloch spaces.

The main objective of this note is to show that the technical developed by Ramos-Fernández et. al. $[15,19,6,5]$, using certain maximal analytic functions, can be used to obtain new characterizations of the continuity and compactness of the weighted composition operators acting on Korenblum and $\alpha$-Bloch spaces. In fact, in Section 2, we use maximal functions in certain log-growth space and in Korenblum spaces to give a new characterization of the continuity and compactness of 
weighted composition operators on these spaces. As consequence of our results in Section 2, in Section 3, we give similar results for $W_{\mathfrak{u}, \phi}$ acting between $\alpha$-Bloch spaces and in Section 4 , we give new characterizations of the continuity and compactness of the composition operators acting between $\alpha$-Zygmund spaces.

\section{The case of weighted composition operators acting on cer- tain growth spaces}

We consider first the case of weighted composition operators $W_{\mathfrak{u}, \phi}$ from $H_{v_{\log }}^{\infty}$ to $H_{\beta}^{\infty}$. Our goal is to find maximal functions $\mathrm{g}_{\mathrm{a}} \in \mathrm{H}_{v_{\log }}^{\infty}$ with $\mathrm{a} \in \mathbb{D}$ in the sense that

$$
\left|g_{a}(a)\right| \geq K \sup _{\|f\|_{H_{v_{\text {log }}^{\infty}}^{\infty}} \leq 1}\{|f(a)|\}
$$

for some constant $\mathrm{K}>0$. Here

$$
\nu_{\log }(z)=\left(\log \left(\frac{e}{1-|z|^{2}}\right)\right)^{-1}
$$

is a weight defined on $\mathbb{D}$ which is radial, typical and non-increasing. The weight $v_{\log }$ is related with the Bloch space $\mathcal{B}$, in fact it is easy to see that $\mathcal{B}$ is continuously contained into $\mathrm{H}_{v_{\log }}^{\infty}$.

A calculation tell us that we have to consider the functions $g_{a}$ defined by

$$
g_{a}(z)=\frac{1-|a|^{2}}{2(1-\bar{a} z)}(1-\log (1-\bar{a} z))
$$

with $z \in \mathbb{D}$, where $\log (w)=\log |w|+\operatorname{iarg}(w)$ denotes the logarithm whose imaginary part lies in the interval $(-\pi, \pi]$, which is holomorphic in the open Euclidean disk with center at 1 and radius 1. Then, following the ideas in [15] and [5], we have obtained the following result:

Theorem 2.1. Let $\beta>0$ be fixed, $\phi: \mathbb{D} \rightarrow \mathbb{D}$ and $u: \mathbb{D} \rightarrow \mathbb{C}$ holomorphic functions. Then

(1) The weighted composition operator $\mathrm{W}_{\mathrm{u}, \phi}$ is continuous from $\mathrm{H}_{v_{\log }}^{\infty}$ to $\mathrm{H}_{\beta}^{\infty}$ if and only if

$$
\sup _{a \in \mathbb{D}}\left\|W_{u, \phi}\left(g_{a}\right)\right\|_{H_{\beta}^{\infty}}<\infty \text {. }
$$

(2) The operator $\mathrm{W}_{\mathfrak{u}, \phi}: \mathrm{H}_{v_{\log }}^{\infty} \rightarrow \mathrm{H}_{\beta}^{\infty}$ is compact if and only if

$$
\lim _{|\mathbf{a}| \rightarrow 1^{-}}\left\|W_{\mathfrak{u}, \phi}\left(g_{\mathfrak{a}}\right)\right\|_{H_{\beta}^{\infty}}=0 .
$$

Proof. Suppose first that $W_{\mathfrak{u}, \phi}: H_{v_{\log }}^{\infty} \rightarrow H_{\beta}^{\infty}$ is continuous. Observe that for each $a \in \mathbb{D}$, we 
have

$$
\begin{aligned}
\left\|g_{\mathrm{a}}\right\|_{H_{v_{\log }}^{\infty}} & =\sup _{z \in \mathbb{D}} \frac{1}{\log \left(\frac{e}{1-|z|^{2}}\right)} \cdot \frac{1-|\mathrm{a}|^{2}}{2|1-\overline{\mathrm{a}} z|}|1-\log (1-\overline{\mathrm{a}} z)| \\
& \leq \sup _{z \in \mathbb{D}} \frac{|1-\log | 1-\overline{\mathrm{a}} z||+|\arg (1-\overline{\mathrm{a}} z)|}{\log \left(\frac{e}{1-|z|^{2}}\right)} \\
& \leq \sup _{z \in \mathbb{D}} \frac{1-\log (1-|z|)+\pi}{\log \left(\frac{e}{1-|z|^{2}}\right)} \\
& \leq \sup _{z \in \mathbb{D}}\left(\frac{\log \left(\frac{e}{1-|z|^{2}}\right)}{\log \left(\frac{e}{1-|z|^{2}}\right)}+\frac{\log (1+|z|)}{\log \left(\frac{e}{1-|z|^{2}}\right)}\right)+\pi \leq 1+\log (2)+\pi .
\end{aligned}
$$

Then, we can see that there exists a constant $\mathrm{K}>0$ such that

$$
\left\|\mathrm{W}_{\mathrm{u}, \phi}\left(\mathrm{g}_{\mathrm{a}}\right)\right\|_{\mathrm{H}_{\beta}^{\infty}} \leq\left\|\mathrm{W}_{\mathrm{u}, \phi}\right\|\left\|\mathrm{g}_{\mathrm{a}}\right\|_{\mathrm{H}_{v_{\log }}} \leq \mathrm{K}\left\|\mathrm{W}_{\mathrm{u}, \phi}\right\|
$$

and (2.3) follows. Conversely, if (2.3) is true, then for each $s \in \mathbb{D}$ we have

$$
\frac{\left(1-|s|^{2}\right)^{\beta}}{v_{\log }(\phi(s))}|u(s)|=2\left(1-|s|^{2}\right)^{\beta}\left|g_{\phi(s)}(\phi(s))\right||u(s)| \leq 2 \sup _{a \in \mathbb{D}}\left\|W_{u, \phi}\left(g_{a}\right)\right\|_{H_{\beta}^{\infty}}
$$

and the item (1) follows from the continuity's theorem of Montes-Rodríguez [16] (see also Contreras and Hernández-Díaz [9]) since the weight $v_{\log }$ is essential.

Now we are going to show the item (2). In virtue of Tjani's lemma in [21] and since $g_{a}$ is a bounded sequence in $\mathrm{H}_{v_{\log }}^{\infty}$ which converges to zero uniformly on compact subsets of $\mathbb{D}$, we conclude that if the operator $W_{u, \phi}: H_{v_{\log }}^{\infty} \rightarrow H_{\beta}^{\infty}$ is compact then the relation (2.4) holds. Conversely, if the relation (2.4) is true, then for every $\varepsilon>0$ we can find $r_{1} \in(1 / 2,1)$ such that $\left\|W_{u, \phi}\left(g_{a}\right)\right\|_{H_{\beta}^{\infty}}<\varepsilon$ whenever $r_{1}<|a|<1$. Hence, if $z \in \mathbb{D}$ satisfies $|\phi(z)|>r_{1}$, then we can write

$$
\begin{aligned}
\frac{\left(1-|z|^{2}\right)^{\beta}}{v_{\log }(\phi(z))}|\mathfrak{u}(z)| & =2\left(1-|z|^{2}\right)^{\beta}\left|g_{\phi(z)}(\phi(z))\right||\mathfrak{u}(z)| \\
& \leq \sup _{w \in \mathbb{D}}\left(1-|w|^{2}\right)^{\beta}\left|g_{\phi(z)}(\phi(w))\right||\mathfrak{u}(w)| \\
& =\left\|W_{\mathfrak{u}, \phi}\left(g_{\phi(z)}\right)\right\|_{H_{\beta}^{\infty}}<\varepsilon .
\end{aligned}
$$

This shows that

$$
\lim _{|\phi(z)| \rightarrow 1^{-}} \frac{\left(1-|z|^{2}\right)^{\beta}}{v_{\log }(\phi(z))}|\mathfrak{u}(z)|=0
$$

and $W_{u, \phi}: H_{v_{\log }}^{\infty} \rightarrow H_{\beta}^{\infty}$ is compact in virtue of a result due to Montes-Rodríguez [16] (see also Contreras and Hernández-Díaz [9]) and the fact that $v_{\log }$ is an essential weight.

Next, we are going to consider the case of the weighted composition operator $W_{\mathfrak{u}, \phi}$ acting from $H_{\alpha}^{\infty}$ to $H_{\beta}^{\infty}$, where $\alpha$ and $\beta$ are positive parameters fixed. We need to find maximal functions $\sigma_{\alpha}^{(\alpha)} \in H_{\alpha}^{\infty}$ in the sense of the relation (2.1), changing, of course, the space $H_{v_{\log }}^{\infty}$ by $H_{\alpha}^{\infty}$. 
In this case, for $a \in \mathbb{D}$, we consider functions $\sigma_{a}^{(\alpha)}$ defined by

$$
\sigma_{a}^{(\alpha)}(z)=\frac{1-|a|}{(1-\bar{a} z)^{\alpha+1}},
$$

where $z \in \mathbb{D}$. Then, the argument in the proof of Theorem 2.1 allow us to show the following result:

Theorem 2.2. Let $\alpha, \beta>0$ be fixed and $u: \mathbb{D} \rightarrow \mathbb{C}, \phi: \mathbb{D} \rightarrow \mathbb{D}$ holomorphic functions.

(1) The weighted composition operators $\mathrm{W}_{\mathbf{u}, \phi}$ is bounded from $\mathrm{H}_{\alpha}^{\infty}$ to $\mathrm{H}_{\beta}^{\infty}$ if and only if

$$
\sup _{\mathbf{a} \in \mathbb{D}}\left\|W_{u, \phi}\left(\sigma_{a}^{(\alpha)}\right)\right\|_{H_{\beta}^{\infty}}<\infty
$$

(2) The weighted composition operators $\mathrm{W}_{\mathfrak{u}, \phi}: \mathrm{H}_{\alpha}^{\infty} \rightarrow \mathrm{H}_{\beta}^{\infty}$ is compact if and only if

$$
\lim _{|\mathbf{a}| \rightarrow 1^{-}}\left\|W_{u, \phi}\left(\sigma_{a}^{(\alpha)}\right)\right\|_{H_{\beta}^{\infty}}=0
$$

Proof. The result follows arguing as in the proof of Theorem 2.1. Indeed, if (2.6) is true, then for any $s \in \mathbb{D}$, we have

$$
\begin{aligned}
\frac{\left(1-|s|^{2}\right)^{\beta}}{\left(1-|\phi(s)|^{2}\right)^{\alpha}}|\mathrm{u}(\mathrm{s})| & =\left(1-|s|^{2}\right)^{\beta}(1+|\phi(s)|)\left|\sigma_{\phi(s)}^{(\alpha)}(\phi(s))\right||u(s)| \\
& \leq 2 \sup _{\mathrm{a} \in \mathbb{D}}\left\|W_{u, \phi}\left(\sigma_{a}^{(\alpha)}\right)\right\|_{H_{\beta}^{\infty}}
\end{aligned}
$$

and the continuity of $W_{u, \phi}: H_{\alpha}^{\infty} \rightarrow H_{\beta}^{\infty}$ follows from the continuity's theorem of Montes-Rodríguez [16] (see also Contreras and Hernández-Díaz [9]). Conversely, if $W_{u, \phi}: H_{\alpha}^{\infty} \rightarrow H_{\beta}^{\infty}$ is continuous, then we have

$$
\begin{aligned}
& \sup _{\mathbf{a} \in \mathbb{D}}\left\|W_{u, \phi}\left(\sigma_{a}^{(\alpha)}\right)\right\|_{H_{\beta}^{\infty}} \leq\left\|W_{u, \phi}\right\| \sup _{a \in \mathbb{D}}\left\|\sigma_{a}^{(\alpha)}\right\|_{H_{\alpha}^{\infty}} \\
& =\left\|W_{\mathfrak{u}, \phi}\right\| \sup _{a \in \mathbb{D}} \sup _{z \in \mathbb{D}}\left(1-|z|^{2}\right)^{\alpha} \frac{1-|a|}{|1-\bar{a} z|^{\alpha+1}} \\
& \leq 2^{\alpha}\left\|W_{u, \phi}\right\| \text {. }
\end{aligned}
$$

This shows the equivalence in the item 1.

Now, since $\sigma_{\mathrm{a}}^{(\alpha)}$ converges to zero uniformly on compact subsets of $\mathbb{D}$ as $|\mathrm{a}| \rightarrow 1^{-}$and $\left\|\sigma_{\mathrm{a}}^{(\alpha)}\right\|_{\mathrm{H}_{\alpha}^{\infty}} \leq 2^{\alpha}$ for all $a \in \mathbb{D}$, Tjani's lemma in [21] implies that if $W_{\mathrm{u}, \phi}: \mathrm{H}_{\alpha}^{\infty} \rightarrow \mathrm{H}_{\beta}^{\infty}$ is compact, then

$$
\lim _{|a| \rightarrow 1^{-}}\left\|W_{u, \phi}\left(\sigma_{a}^{(\alpha)}\right)\right\|_{H_{\beta}^{\infty}}=0 .
$$


Conversely, if the relation (2.7) is true, then for every $\varepsilon>0$ we can find $r_{1} \in(1 / 2,1)$ such that $\left\|W_{\mathfrak{u}, \phi}\left(\sigma_{a}^{(\alpha)}\right)\right\|_{H_{\beta}^{\infty}}<\varepsilon$ whenever $r_{1}<|a|<1$. Hence, if $z \in \mathbb{D}$ satisfies $|\phi(z)|>r_{1}$, then we can write

$$
\begin{aligned}
\frac{\left(1-|z|^{2}\right)^{\beta}}{\left(1-|\phi(z)|^{2}\right)^{\alpha}}|\mathfrak{u}(z)| & =2\left(1-|z|^{2}\right)^{\beta}\left|\sigma_{\phi(z)}^{(\alpha)}(\phi(z))\right||\mathfrak{u}(z)| \\
& \leq 2 \sup _{w \in \mathbb{D}}\left(1-|w|^{2}\right)^{\beta}\left|\sigma_{\phi(z)}^{(\alpha)}(\phi(w))\right||u(w)| \\
& =\left\|W_{\mathfrak{u}, \phi}\left(\sigma_{\phi(z)}^{(\alpha)}\right)\right\|_{H_{\beta}^{\infty}}<\varepsilon .
\end{aligned}
$$

This shows that

$$
\lim _{|\phi(z)| \rightarrow 1^{-}} \frac{\left(1-|z|^{2}\right)^{\beta}}{\left(1-|\phi(z)|^{2}\right)^{\alpha}}|u(z)|=0
$$

and $W_{\mathfrak{u}, \phi}: H_{\alpha}^{\infty} \rightarrow H_{\beta}^{\infty}$ is compact in virtue of a result due to Montes-Rodríguez [16] (see also Contreras and Hernández-Díaz [9]).

As a consequence of our results, we obtain a recent result about the continuity and compactness of composition operators acting between $\alpha$-Bloch spaces due to Malavé and Ramos-Fernández [15]. For $a \in \mathbb{D}$ we set

$$
\lambda_{\mathrm{a}}^{(\alpha)}(z)=\alpha \overline{\mathrm{a}} \int_{0}^{z} \sigma_{\mathrm{a}}^{(\alpha)}(\mathrm{s}) \mathrm{d} s=(1-|\mathrm{a}|)\left[\frac{1}{(1-\overline{\mathrm{a}} z)^{\alpha}}-1\right] .
$$

Then we have the following result:

Corolary 1. Let $\alpha, \beta>0$ be fixed and $\phi: \mathbb{D} \rightarrow \mathbb{D}$ an holomorphic function. Then

(1) The operator $\mathrm{C}_{\phi}$ from $\mathcal{B}^{\alpha}$ to $\mathcal{B}^{\beta}$ is bounded if and only if

$$
\sup _{\mathbf{a} \in \mathbb{D}}\left\|C_{\phi}\left(\lambda_{a}^{(\alpha)}\right)\right\|_{\mathcal{B}^{\beta}}<\infty \text {. }
$$

(2) The operator $\mathrm{C}_{\phi}: \mathcal{B}^{\alpha} \rightarrow \mathcal{B}^{\beta}$ is compact if and only if

$$
\lim _{|\mathrm{a}| \rightarrow 1^{-}}\left\|\mathrm{C}_{\phi}\left(\lambda_{\mathrm{a}}^{(\alpha)}\right)\right\|_{\mathcal{B}^{\beta}}=0 .
$$

Proof. This result follows from the fact that $C_{\phi}: \mathcal{B}^{\alpha} \rightarrow \mathcal{B}^{\beta}$ is continuous (resp. compact) if and only if $W_{\phi^{\prime}, \phi}: \mathrm{H}_{\alpha}^{\infty} \rightarrow \mathrm{H}_{\beta}^{\infty}$ is continuous (resp. compact).

\section{Application. A new criterion for the continuity and com- pactness of weighted composition operators acting be- tween $\alpha$-Bloch spaces}

Now, we are going to use the functions $\sigma_{a}^{(\alpha)}, \lambda_{a}^{(\alpha)}$ and $g_{a}$ defined in (2.5), (2.8) and (2.2) to characterize the continuity and compactness of the weighted composition operator $W_{\mathfrak{u}, \phi}$ acting between 
$\alpha$-Bloch spaces. According to the continuity's results in [17, Theorem 2.1] and [16, Theorem 2.1] (see also [9, Proposition 3.1]) we arrive to the following result:

Theorem 3.1. Let $\alpha, \beta>0$ fixed, $\phi: \mathbb{D} \rightarrow \mathbb{D}$ and $u: \mathbb{D} \rightarrow \mathbb{C}$ holomorphic functions. Then

(1) If $0<\alpha<1$, then the operator $W_{u, \phi}$ from $\mathcal{B}^{\alpha}$ into $\mathcal{B}^{\beta}$ is continuous if and only if $u \in \mathcal{B}^{\beta}$ and

$$
\sup _{a \in \mathbb{D}}\left\|I_{\mathfrak{u}} C_{\phi}\left(\lambda_{a}^{(\alpha)}\right)\right\|_{\mathcal{B}^{\beta}}<\infty .
$$

(2) If $\alpha=1$, the operator $W_{\mathfrak{u}, \phi}$ from $\mathcal{B}$ into $\mathcal{B}^{\beta}$ is continuous if and only if

$$
\sup _{\mathrm{a} \in \mathbb{D}}\left\|J_{\mathfrak{u}} C_{\phi}\left(g_{a}\right)\right\|_{\mathcal{B}^{\beta}}<\infty \text { and } \sup _{a \in \mathbb{D}}\left\|\mathrm{I}_{\mathfrak{u}} C_{\phi}\left(\lambda_{a}^{(1)}\right)\right\|_{\mathcal{B}^{\beta}}<\infty .
$$

(3) If $\alpha>1$, then the operator $\mathrm{W}_{\mathfrak{u}, \phi}$ from $\mathcal{B}^{\alpha}$ into $\mathcal{B}^{\beta}$ is continuous if and only if

$$
\sup _{a \in \mathbb{D}}\left\|J_{\mathcal{u}} C_{\phi}\left(\sigma_{a}^{(\alpha-1)}\right)\right\|_{\mathcal{B}^{\beta}}<\infty \text { and } \sup _{a \in \mathbb{D}}\left\|I_{\mathcal{u}} C_{\phi}\left(\lambda_{a}^{(\alpha)}\right)\right\|_{\mathcal{B}^{\beta}}<\infty .
$$

Where for $\mathrm{u} \in \mathrm{H}(\mathbb{D})$ fixed and $\mathbf{f} \in \mathrm{H}(\mathbb{D})$, we define the operators $\mathrm{I}_{\mathfrak{u}}(\mathbf{f})$ and $\mathrm{J}_{\mathfrak{u}}(\mathbf{f})$ by

$$
\mathrm{I}_{\mathfrak{u}}(f)(z)=\int_{0}^{z} f^{\prime}(w) \mathfrak{u}(w) \mathrm{d} w \quad \text { and } \quad \mathrm{J}_{\mathfrak{u}}(f)(z)=\int_{0}^{z} f(w) \mathfrak{u}^{\prime}(w) \mathrm{d} w
$$

with $z \in \mathbb{D}$.

Example 1. If $u \equiv 1$, then $J_{u}$ is the null operator and we obtain that $C_{\phi}: \mathcal{B}^{\alpha} \rightarrow \mathcal{B}^{\beta}$ is continuous if and only if

$$
\sup _{a \in \mathbb{D}}\left\|C_{\phi}\left(\lambda_{a}^{(\alpha)}\right)\right\|_{\mathcal{B}^{\beta}}<\infty .
$$

Again, we have extended a result in [15].

The same argument in the paragraph before Theorem 3.1 allow us to show a result about the compactness of the weighted composition operators $W_{\mathfrak{u}, \phi}$ acting between $\alpha$-Bloch spaces. In fact, by [17, Theorem 3.1], [16, Theorem 2.1] (or [9, Corollary 4.3]) and the item 2 in our Theorem 2.1 and Theorem 2.2, imply the following result:

Theorem 3.2. Let $\alpha, \beta>0$ be fixed, $\phi: \mathbb{D} \rightarrow \mathbb{D}$ and $u: \mathbb{D} \rightarrow \mathbb{C}$ holomorphic functions. Then

(1) If $0<\alpha<1$, then $\mathrm{W}_{\mathfrak{u}, \phi}: \mathcal{B}^{\alpha} \rightarrow \mathcal{B}^{\beta}$ is compact if and only if $\boldsymbol{u} \in \mathcal{B}^{\beta}$ and

$$
\lim _{|\mathbf{a}| \rightarrow 1^{-}}\left\|\mathrm{I}_{\mathrm{u}} \mathrm{C}_{\phi}\left(\lambda_{\mathrm{a}}^{(\alpha)}\right)\right\|_{\mathcal{B}^{\beta}}=0 .
$$

(2) If $\alpha=1$, then $\mathrm{W}_{\mathrm{u}, \varphi}: \mathcal{B} \rightarrow \mathcal{B}^{\beta}$ is compact if and only if

$$
\lim _{|\mathbf{a}| \rightarrow 1^{-}}\left\|J_{\mathfrak{u}} C_{\phi}\left(g_{a}\right)\right\|_{\mathcal{B}^{\beta}}=0 \text { and } \lim _{|\mathbf{a}| \rightarrow 1^{-i}}\left\|I_{\mathcal{u}^{\prime}} C_{\phi}\left(\lambda_{a}^{(1)}\right)\right\|_{\mathcal{B}^{\beta}}=0 \text {. }
$$


(3) If $\alpha>1$, then $\mathrm{W}_{\mathrm{u}, \phi}: \mathcal{B}^{\alpha} \rightarrow \mathcal{B}^{\beta}$ is compact if and only if

$$
\lim _{|\mathbf{a}| \rightarrow 1^{-}}\left\|J_{\mathcal{u}} C_{\phi}\left(\sigma_{a}^{(\alpha-1)}\right)\right\|_{\mathcal{B}^{\beta}}=0, \text { and } \lim _{|\mathbf{a}| \rightarrow 1^{-}}\left\|I_{\mathcal{U}^{\mathcal{L}}} C_{\phi}\left(\lambda_{a}^{(\alpha)}\right)\right\|_{\mathcal{B}^{\beta}}=0 \text {. }
$$

Example 2. If $u \equiv 1$, then we have that the composition operator $C_{\phi}: \mathcal{B}^{\alpha} \rightarrow \mathcal{B}^{\beta}$ is compact if and only if

$$
\lim _{|\mathbf{a}| \rightarrow 1^{-}}\left\|C_{\phi}\left(\lambda_{\mathrm{a}}^{(\alpha)}\right)\right\|_{\mathcal{B}^{\beta}}=0 \text {. }
$$

This extend a result in [15].

\section{Application. Continuity and compactness of the compo- sition operators acting between $\alpha$-Zygmund spaces}

For $\alpha>0$ fixed, the $\alpha$-Zygmund space, denoted by $\mathcal{Z}_{\alpha}$, consist of all holomorphic functions $f$ on $\mathbb{D}$ such that

$$
\|f\|_{\widetilde{\mathcal{Z}}_{\alpha}}:=\sup _{z \in \mathbb{D}}\left(1-|z|^{2}\right)^{\alpha}\left|f^{\prime \prime}(z)\right|<\infty .
$$

Clearly, $f \in \mathcal{Z}_{\alpha}$ if and only if $f^{\prime} \in \mathcal{B}^{\alpha}$. Also, it is easy to see that $\mathcal{Z}_{\alpha}$ is a Banach space with the norm

$$
\|f\|_{\mathcal{Z}_{\alpha}}=|f(0)|+\left|f^{\prime}(0)\right|+\|f\|_{\tilde{\mathcal{Z}}_{\alpha}}=|f(0)|+\left\|f^{\prime}\right\|_{\mathcal{B}^{\alpha}} .
$$

We will use our results in the above sections to characterize the continuity and the compactness of the composition operator $\mathrm{C}_{\phi}$ acting between $\alpha$-Zygmund spaces in terms of the composition of certain special functions in $\mathcal{Z}_{\alpha}$. The key of our result repose in the following lemma:

Lemma 4.1. Let $\alpha, \beta>0$ be fixed and $\phi: \mathbb{D} \rightarrow \mathbb{D}$ an holomorphic function. Then

(1) The operator $C_{\phi}$ from $\mathcal{Z}_{\alpha}$ to $\mathcal{Z}_{\beta}$ is bounded if and only if $W_{\phi^{\prime}, \phi}: \mathcal{B}^{\alpha} \rightarrow \mathcal{B}^{\beta}$ is bounded.

(2) The operator $\mathrm{C}_{\phi}: \mathcal{Z}_{\alpha} \rightarrow \mathcal{Z}_{\beta}$ is compact if and only if $\mathrm{W}_{\phi^{\prime}, \phi}: \mathcal{B}^{\alpha} \rightarrow \mathcal{B}^{\beta}$ is compact.

Proof. Suppose first that the operator $\mathrm{C}_{\phi}: \mathcal{Z}_{\alpha} \rightarrow \mathcal{Z}_{\beta}$ is bounded. There exists a constant $\mathrm{L}>0$ such that $\left\|C_{\phi}(g)\right\|_{\mathcal{Z}_{\beta}} \leq \mathrm{L}\|g\|_{\mathcal{Z}_{\alpha}}$ for all $g \in \mathcal{Z}_{\alpha}$. Thus, for any $\mathrm{f} \in \mathcal{B}^{\alpha}$, we have that

$$
g(z)=\int_{0}^{z} f(s) d s
$$

belongs to $\mathcal{Z}_{\alpha}$ and therefore,

$$
\begin{aligned}
\left\|W_{\phi^{\prime}, \phi}(f)\right\|_{\mathcal{B}^{\beta}} & =\left\|\phi^{\prime} \cdot f \circ \phi\right\|_{\mathcal{B}^{\beta}}=\left\|\phi^{\prime} \cdot g^{\prime} \circ \phi\right\|_{\mathcal{B}^{\beta}}=\left\|(g \circ \phi)^{\prime}\right\|_{\mathcal{B}^{\beta}} \\
& \leq|(g \circ \phi)(0)|+\left\|(g \circ \phi)^{\prime}\right\|_{\mathcal{B}^{\beta}}=\left\|C_{\phi}(g)\right\|_{\mathcal{Z}_{\beta}} \leq \mathrm{L}\|g\|_{\mathcal{Z}_{\alpha}}=\mathrm{L}\|f\|_{\mathcal{B}^{\alpha}}
\end{aligned}
$$

since $g(0)=0$. This shows that $W_{\Phi^{\prime}, \phi}: \mathcal{B}^{\alpha} \rightarrow \mathcal{B}^{\beta}$ is bounded. 
Conversely, if $W_{\phi^{\prime}, \phi}: \mathcal{B}^{\alpha} \rightarrow \mathcal{B}^{\beta}$ is bounded, then there exists a constant $\mathrm{L}>0$ such that $\left\|W_{\phi^{\prime}, \phi}(g)\right\|_{\mathcal{B}^{\beta}} \leq \mathrm{L}\|g\|_{\mathcal{B}^{\alpha}}$ for all $\mathrm{g} \in \mathcal{B}^{\alpha}$. Thus, for any $\mathrm{f} \in \mathcal{Z}_{\alpha}$, we have that $\mathrm{g}=\mathrm{f}^{\prime} \in \mathcal{B}^{\alpha}$ and hence

$$
\left\|(f \circ \phi)^{\prime}\right\|_{\mathcal{B}^{\beta}}=\left\|W_{\phi^{\prime}, \phi}(g)\right\|_{\mathcal{B}^{\beta}} \leq \mathrm{L}\left\|f^{\prime}\right\|_{\mathcal{B}^{\alpha}} .
$$

Furthermore, since $f \in H(\mathbb{D})$, we can write

$$
|f(\phi(0))| \leq|f(0)|+\left|f^{\prime}(0)\right|+\int_{0}^{\phi(0)} \int_{0}^{s}\left|f^{\prime \prime}(w)\right||d w||d s| .
$$

Therefore, multiplying and dividing by $\left(1-|w|^{2}\right)^{\alpha}$ inside the integral symbol, we can find a constant $\mathrm{K}>0$, which can depend on $\phi(0)$, such that $|\mathrm{f}(\phi(0))| \leq \mathrm{K}\|\mathrm{f}\|_{\mathcal{Z}_{\alpha}}$. We conclude then that

$$
\left\|C_{\phi}(f)\right\|_{\mathcal{Z}_{\beta}}=|f(\phi(0))|+\left\|(f \circ \phi)^{\prime}\right\|_{\mathcal{B}^{\beta}} \leq(K+L)\|f\|_{\mathcal{Z}_{\alpha}}
$$

and the operator $C_{\phi}: \mathcal{Z}_{\alpha} \rightarrow \mathcal{Z}_{\beta}$ is bounded. This shows the item (1).

Now we are going to show the item (2). Suppose first that $W_{\Phi^{\prime}, \phi}: \mathcal{B}^{\alpha} \rightarrow \mathcal{B}^{\beta}$ is a compact operator and let $\left\{g_{n}\right\}$ be a bounded sequence in $\mathcal{Z}_{\alpha}$, then the sequence $\left\{g_{n}^{\prime}\right\}$ is a bounded sequence in $\mathcal{B}^{\alpha}$. Hence, by passing to a subsequence, we can suppose that $\left\{\mathbf{W}_{\phi^{\prime}, \phi}\left(\mathbf{g}_{\mathfrak{n}}^{\prime}\right)\right\}$ converges in $\mathcal{B}^{\beta}$ to a function $h \in \mathcal{B}^{\beta}$. Also, $\left\{g_{\mathfrak{n}}(\phi(0))\right\}$ is a bounded sequence in $\mathbb{C}$ and, by passing to a subsequence, we can suppose that $\left\{g_{\mathfrak{n}}(\phi(0))\right\}$ goes to $z_{0} \in \mathbb{C}$ as $n \rightarrow \infty$. Then, we can define the function

$$
g(z)=z_{0}+\int_{0}^{z} h(s) d s
$$

which belongs to $\mathcal{Z}_{\beta}$ and we have that

$$
\left\|C_{\phi}\left(g_{n}\right)-g\right\|_{\mathcal{Z}_{\beta}}=\left|g_{\mathfrak{n}}(\phi(0))-g(0)\right|+\left\|W_{\phi^{\prime}, \phi}\left(g_{n}^{\prime}\right)-h\right\|_{\mathcal{B}^{\beta}} \rightarrow 0
$$

as $n \rightarrow \infty$. That is, $\mathrm{C}_{\phi}: \mathcal{Z}_{\alpha} \rightarrow \mathcal{Z}_{\beta}$ is a compact operator.

Conversely, if $C_{\phi}: \mathcal{Z}_{\alpha} \rightarrow \mathcal{Z}_{\beta}$ is a compact operator and $\left\{f_{n}\right\}$ is a bounded sequence in $\mathcal{B}^{\alpha}$, then the sequence $\left\{g_{n}\right\}$ defined by

$$
g_{n}(z)=\int_{0}^{z} f_{n}(s) d s
$$

is bounded in $\mathcal{Z}_{\alpha}$ and hence, by passing to a subsequence, we can suppose that $\left\{\mathrm{C}_{\phi}\left(\mathrm{g}_{\mathrm{n}}\right)\right\}$ converges in $\mathcal{Z}_{\beta}$ to a function $h \in \mathcal{Z}_{\beta}$. Therefore, we can write

$$
\begin{aligned}
\left\|W_{\phi^{\prime}, \phi}\left(f_{n}\right)-h^{\prime}\right\|_{\mathcal{B}^{\beta}} & =\left\|W_{\phi^{\prime}, \phi}\left(g_{n}^{\prime}\right)-h^{\prime}\right\|_{\mathcal{B}^{\beta}} \\
& =\left\|\left(C_{\phi}\left(g_{n}\right)-h\right)^{\prime}\right\|_{\mathcal{B}^{\beta}} \\
& \leq\left\|C_{\phi}\left(g_{n}\right)-h\right\|_{\mathcal{Z}_{\beta}} \rightarrow 0
\end{aligned}
$$

as $\mathrm{n} \rightarrow \infty$ and the result follows since $\mathrm{h}^{\prime} \in \mathcal{B}^{\beta}$.

As consequence of above lemma, [17, Theorem 2.1], [16, Theorem 2.1] (or [9, Proposition 3.1]) and our Theorem 2.1, Theorem 2.2 and Theorem 3.1, we have: 
Corolary 2. Let $\alpha, \beta>0$ be fixed and $\phi: \mathbb{D} \rightarrow \mathbb{D}$ an holomorphic function. Then, for each case, the following propositions are equivalents:

(1) Case $0<\alpha<1$.

(a) The operator $\mathrm{C}_{\phi}$ from $\mathcal{Z}_{\alpha}$ to $\mathcal{Z}_{\beta}$ is continuous,

(b) $\phi \in \mathcal{Z}_{\beta}$ and $\mathrm{W}_{\left(\phi^{\prime}\right)^{2}, \phi}: \mathrm{H}_{\alpha}^{\infty} \rightarrow \mathrm{H}_{\beta}^{\infty}$ is continuous,

(c) $\phi \in \mathcal{Z}_{\beta}$ and

$$
\sup _{w \in \mathbb{D}} \frac{\left(1-|w|^{2}\right)^{\beta}}{\left(1-|\phi(w)|^{2}\right)^{\alpha}}\left|\phi^{\prime}(w)\right|^{2}<\infty
$$

(d) $\phi \in \mathcal{Z}_{\beta}$ and

$$
\sup _{\mathbf{a} \in \mathbb{D}}\left\|W_{\left(\phi^{\prime}\right)^{2}, \phi}\left(\sigma_{a}^{(\alpha)}\right)\right\|_{H_{\beta}^{\infty}}<\infty
$$

(e) $\phi \in \mathcal{Z}_{\beta}$

$$
\sup _{\mathbf{a} \in \mathbb{D}}\left\|\mathrm{I}_{\phi^{\prime}} \mathrm{C}_{\phi}\left(\lambda_{\mathrm{a}}^{(\alpha)}\right)\right\|_{\mathcal{B}^{\beta}}<\infty
$$

(2) Case $\alpha=1$.

(a) The operator $\mathrm{C}_{\phi}$ from $\mathcal{Z}_{\alpha}$ to $\mathcal{Z}_{\beta}$ is continuous,

(b) $\mathrm{W}_{\phi^{\prime \prime}, \phi}: \mathrm{H}_{v_{\log }}^{\infty} \rightarrow \mathrm{H}_{\beta}^{\infty}$ and $\mathrm{W}_{\left(\phi^{\prime}\right)^{2}, \phi}: \mathrm{H}_{1}^{\infty} \rightarrow \mathrm{H}_{\beta}^{\infty}$ are continuous,

(c) $\sup _{w \in \mathbb{D}} \frac{\left(1-|w|^{2}\right)^{\beta}}{v_{\log }(\phi(w))}\left|\phi^{\prime \prime}(w)\right|<\infty$, and $\sup _{w \in \mathbb{D}} \frac{\left(1-|w|^{2}\right)^{\beta}}{1-|\phi(w)|^{2}}\left|\phi^{\prime}(w)\right|^{2}<\infty$.

(d) $\sup _{\mathbf{a} \in \mathbb{D}}\left\|W_{\phi^{\prime \prime}, \phi}\left(g_{a}\right)\right\|_{H_{\beta}^{\infty}}<\infty$ and $\sup _{\mathbf{a} \in \mathbb{D}}\left\|W_{\left(\phi^{\prime}\right)^{2}, \phi}\left(\sigma_{a}^{(1)}\right)\right\|_{H_{\beta}^{\infty}}<\infty$

(e) $\sup _{\mathbf{a} \in \mathbb{D}}\left\|J_{\phi^{\prime}} \mathrm{C}_{\phi}\left(g_{\mathrm{a}}\right)\right\|_{\mathcal{B}^{\beta}}<\infty$ and $\sup _{\mathrm{a} \in \mathbb{D}}\left\|\mathrm{I}_{\phi^{\prime}} \mathrm{C}_{\phi}\left(\lambda_{\mathrm{a}}^{(1)}\right)\right\|_{\mathcal{B}^{\beta}}<\infty$.

(3) Case $\alpha>1$.

(a) The operator $\mathrm{C}_{\phi}$ from $\mathcal{Z}_{\alpha}$ to $\mathcal{Z}_{\beta}$ is continuous,

(b) $\mathrm{W}_{\phi^{\prime \prime}, \phi}: \mathrm{H}_{\alpha-1}^{\infty} \rightarrow \mathrm{H}_{\beta}^{\infty}$ and $\mathrm{W}_{\left(\phi^{\prime}\right)^{2}, \phi}: \mathrm{H}_{\alpha}^{\infty} \rightarrow \mathrm{H}_{\beta}^{\infty}$ are continuous,

(c) $\sup _{w \in \mathbb{D}} \frac{\left(1-|w|^{2}\right)^{\beta}}{\left(1-|\phi(w)|^{2}\right)^{\alpha-1}}\left|\phi^{\prime \prime}(w)\right|<\infty, \quad$ and $\sup _{w \in \mathbb{D}} \frac{\left(1-|w|^{2}\right)^{\beta}}{\left(1-|\phi(w)|^{2}\right)^{\alpha}}\left|\phi^{\prime}(w)\right|^{2}<\infty$.

(d) $\sup _{\mathbf{a} \in \mathbb{D}}\left\|W_{\phi^{\prime \prime}, \phi}\left(\sigma_{\mathrm{a}}^{(\alpha-1)}\right)\right\|_{\mathrm{H}_{\beta}^{\infty}}<\infty$ and $\sup _{\mathbf{a} \in \mathbb{D}}\left\|W_{\left(\phi^{\prime}\right)^{2}, \phi}\left(\sigma_{\mathrm{a}}^{(\alpha)}\right)\right\|_{\mathrm{H}_{\beta}^{\infty}}<\infty$

(e) $\sup _{\mathrm{a} \in \mathbb{D}}\left\|\mathrm{J}_{\phi^{\prime}} \mathrm{C}_{\phi}\left(\sigma_{\mathrm{a}}^{(\alpha-1)}\right)\right\|_{\mathcal{B}^{\beta}}<\infty$ and $\sup _{\mathrm{a} \in \mathbb{D}}\left\|\mathrm{I}_{\phi^{\prime}} \mathrm{C}_{\phi}\left(\lambda_{\mathrm{a}}^{(\alpha)}\right)\right\|_{\mathcal{B}^{\beta}}<\infty$.

Similar results can be found for the compactness of the operator $\mathrm{C}_{\phi}$ from $\mathcal{Z}_{\alpha}$ to $\mathcal{Z}_{\beta}$. Also, it is possible to write a characterizations using the Zygmund's norm and the fact that $\|\mathrm{f}\|_{\mathcal{Z}_{\alpha}}=$ $|\mathbf{f}(0)|+\left\|\mathbf{f}^{\prime}\right\|_{\mathcal{B}^{\alpha}}$. Here, we can use the operators

$$
\widetilde{\mathrm{I}}_{\mathfrak{u}} f(z)=\int_{0}^{z} I_{\mathfrak{u}}(f(s)) d s \quad \text { and } \quad \widetilde{J}_{\mathfrak{u}} f(z)=\int_{0}^{z} J_{\mathfrak{u}}(f(s)) d s .
$$


Acknowledgement. The authors would like to thank the referees for their valuable comments which helped to improve the manuscript.

\section{References}

[1] J. M. Anderson and J. Duncan, Duals of Banach spaces of entire functions. Glasgow Math. J. 32 (1990), no. 2, 215-220.

[2] K. D. Bierstedt, J. Bonet and J. Taskinen, Associated weights and spaces of holomorphic functions. Studia Math. 127 (1998), 137-168.

[3] K. D. Bierstedt, R. Meise and W. H. Summers, A projective description of weighted inductive limits. Trans. Amer. Math. Soc. 272 (1982), 107-160.

[4] J. Bonet, P. Domański and M. Lindström, Essential norm and weak compactness of composition operators on weighted Banach spaces of analytic function. Canad. Math. Bull. 42 (1999), 139-148.

[5] R. E. Castillo, C. E. Marrero-Rodríguez and J. C. Ramos-Fernández, On a Criterion for Continuity and Compactness of Composition Operators on the Weighted Bloch Space. Mediterr. J. Math. 12, No. 3, pp. 1047-1058.

[6] R. E. Castillo, J. C. Ramos-Fernández and E. M. Rojas, A new essential norm estimate of composition operators from weighted Bloch space into $\mu$-Bloch spaces. J. Funct. Spaces Appl. 2013, Art. ID 817278, 5 pp.

[7] F. Colonna, New criteria for boundedness and compactness of weighted composition operators mapping into the Bloch space. Cent. Eur. J. Math. 11 (2013), no. 1, 55-73.

[8] F. Colonna and S. Li, Weighted composition operators from Hardy spaces into logarithmic Bloch spaces. J. Funct. Spaces Appl. 2012, Art. ID 454820, 20 pp.

[9] M. Contreras and A. Hernández-Díaz, Weighted composition operators in weighted Banach spaces of analytic functions. J. Austral. Math. Soc. Ser. A, 69 (2000), 41-60.

[10] C. C. Cowen and B. D. MacCluer, Composition Operators on Spaces of Analytic Functions, CRC Press, Boca Raton, 1995.

[11] J. Giménez, R. Malavé and J. C. Ramos-Fernández, Composition operators on $\mu$-Bloch type spaces. Rend. Circ. Mat. Palermo, 59 (2010), 107-119.

[12] H. Hedenmalm, B. Korenblum and K. Zhu, Theory of Bergman spaces. Graduate Texts in Mathematics, 199. Springer-Verlag, New York, 2000.

[13] O. Hyvärinen and M. Lindström, Estimates of essential norms of weighted composition operators between Bloch-type spaces. J. Math. Anal. Appl. 393 (2012), 38-44. 
[14] K. Madigan and A. Matheson, Compact composition operators on the Bloch space. Trans. Amer. Math. Soc. 347, (1995), 2679-2687.

[15] M. T. Malavé-Ramírez and J. C. Ramos-Fernández, On a criterion for continuity and compactness of composition operators acting on $\alpha$-Bloch spaces. C. R. Math. Acad. Sci. Paris, Ser. I, 351 (2013), 23-26.

[16] A. Montes-Rodríguez, Weighted composition operators on weighted Banach spaces of analytic functions. J. London Math. Soc. 61 (2000), no. 2, 872-884.

[17] S. Ohno, K. Stroethoff y R. Zhao, Weighted composition operators between Bloch-type spaces. Rocky Mountain J. Math. 33 (2003), no. 1, 191-215.

[18] J. C. Ramos-Fernández, Composition operators between $\mu$-Bloch spaces. Extracta Math. 26 (2011), no. 1, 75-88.

[19] J. C. Ramos-Fernández, A new essential norm estimate of composition operators from $\alpha$-Bloch spaces into $\mu$-Bloch spaces. Internat. J. Math. 24 (2013), no. 14, 1350104, 7 pp.

[20] J. H. Shapiro, Composition Operators and Classical Function Theory, Springer-Verlag, New York, 1993.

[21] M. Tjani, Compact composition operators on some Möbius invariant Banach space. Thesis (Ph.D.)Michigan State University. ProQuest LLC, Ann Arbor, MI, 1996. 68 pp. ISBN: 9780591-27288-8.

[22] M. Tjani, Compact composition operators on Besov spaces. Trans. Amer. Math. Soc. 355, (2003), no. 11, 4683-4698.

[23] K. Zhu, Operator theory in function spaces. Marcel Dekker. New York, 1990.

[24] K. Zhu, Bloch type spaces of analytic functions. Rocky Mountain J. Math. 23 (1993), 11431177. 